\title{
Laser Applications for Estimation of Microbial Activity and Investigation of NIR Skin Autofluorescence
}

\author{
Alexey Lihachev ${ }^{1}$, Dmitrijs Bḷizṇuks ${ }^{2}$, Jānis Liepiṇš ${ }^{3}$, and Ilze Lihacova ${ }^{1}$ \\ ${ }^{1}$ University of Latvia, Institute of Atomic Physics and Spectroscopy, Biophotonics Laboratory, 3 Jelgavas str., \\ Riga LV-1004, Latvia \\ ${ }^{2}$ Riga Technical University, Faculty of Computer Science and Information Technology, 10 Zunda krastmala, \\ Riga LV-1050, Latvia \\ ${ }^{3}$ University of Latvia, Institute of Microbiology and Biotechnology, 1 Jelgavas str., Riga LV-1004, Latvia \\ * e-mail: aleksejs.lihacovs@lu.lv
}

\begin{abstract}
The presented paper demonstrates the ongoing results of the joint research of University of Latvia and Riga Technical University aimed at the development of new laser based optical methods and technologies for fast evaluation of microbial activity in solid media. The second part of the paper represents the results of joint research conducted together with Katya Borisova during our visit to the Institute of Electronics, Bulgarian Academy of Sciences (Bulgaria, Sofia). (C) 2021 Journal of Biomedical Photonics \& Engineering.
\end{abstract}

Keywords: laser speckles; microorganisms; near infrared skin autofluorescence; melanoma cells.

Paper \#3454 received 23 Aug 2021; revised manuscript received 20 Sep 2021; accepted for publication 23 Sep 2021; published online 4 Nov 2021. doi: 10.18287/JBPE21.07.040305.

\section{Introduction}

\subsection{Laser speckle imaging for microorganisms' growth analysis in solid media}

Enumeration of live microbial cells in the sample is one of the basic quantitative measurements in microbiology. Currently counting microorganisms in the early stages of colony formation is possible only by microscope, which is limited to small sample areas. Also, determination of colony size increase dynamics (microorganism growth speed estimation) is an extremely labor-intensive task. Automated counting is possible only by using regular cameras that are limited in optical resolution and therefore are capable of detecting signs of microbial colonies only after a certain size threshold is reached (minimal diameter $0.2 \mathrm{~mm}$ and / or 8-22 h). Therefore, the development of new cost-effective methods for in situ estimation of microorganisms' activity to reduce detection time and labor expenses raise a great practical interest for the scientists and technology developers. One of the options for the fast and early evaluation of microorganisms' activity is a non-contact optical technique called laser speckle contrast imaging. Laser speckle is an interference pattern produced by coherent light reflected or scattered from different parts of the illuminated surface. In the case of non-moving, static scatters, the scattered light generates constant laser speckle patterns. However, if scatterers are spontaneously moving (e.g., Brownian motion, bacteria doubling and chemotaxis) such as particles in a fluid, the individual speckle appears to be "twinkle" or "boiling". This phenomenon is called a "time-varying speckle". Regarding this phenomenon, the laser speckle imaging technique has been proved to monitor moving particles in optically inhomogeneous media by analyzing timevarying laser speckle patterns. Thereby, the analysis of laser speckles patterns provides a powerful opportunity for quantifying the movement activity at the micro and macroscale. Literature data points to the significant progress and potential applications of laser speckle imaging technique to evaluate the dynamic processes in microbiological media. Technology using speckle decorrelation time maps has been demonstrated for the detection of E. coli and B. cereus on a meat (chicken breast) [1]. Speckle analysis has also been applied to biomass growth kinetic measurements in liquid culture, characterization of Colony Forming Units (CFU) morphology, determination of antibiotic susceptibility [2-5]. As well as laser speckle imaging techniques in combination with Deep Learning (DL) and Artificial Neural Networks (ANN) have demonstrated a fast system response of antibacterial susceptibility evaluation in MIC (Minimum Inhibitory Concentration) tests [6]. In the presented paper, we will prove that dynamic laser speckle 
image analysis methods can be used to detect and predict the CFU of different microbes in solid media.

\subsection{Investigation of Near Infrared Autofluorescence under continuous laser excitation}

The phenomenon of autofluorescence in the near infrared region (NIR AF) under continuous laser excitation was described by many authors. The first mention of NIR AF was described by Z. Huang et al. [8] where the NIR AF was analyzed during Raman spectroscopy investigations under $785 \mathrm{~nm}$ excitation. Authors demonstrate promising sensitivity of NIR AF intensity analysis for evaluation of pigmented lesions, as well as, demonstrated applications for melanoma diagnostics. The physical explanation of the NIR AF and its origin are still under discussion, mainly it can be described by absence of absorption spectra of main tissue fluorophores in the NIR region. Several hypotheses describing this phenomenon are discussed in literature, mainly attributing NIR AF to the melanin, endogenous porphyrins and lipofuscin [9-11]. Analysis and detection of NIR AF is a challenging task, since the NIR AF intensity usually is very weak and requires a high sensitivity detecting system equipped with high $>8$ OD filters. The artefacts caused by reflected laser light significantly complicate the adequate detection of the NIR $\mathrm{AF}$ from living tissues. In presented research we have tried to investigate the phenomenon of NIR AF. The experiments were conducted in close collaboration with Ekaterina Borisova during our secondment to the Institute of Electronics, Bulgarian Academy of Sciences. The obtained results and discussion are described below.

\section{Material and methods}

\subsection{Laser speckle imaging system}

The system was assembled for burst capturing of macro scale images under white light and laser illumination. The optical measurement system consists of a laser source, white light source LED, 35mm CS lens@F18, optical attenuator, a testing agar plate (with inoculated bacteria) and a CMOS camera (Fig. 1). The laser speckles were generated by a linearly polarized $635 \mathrm{~nm}$ diode pumped solid state laser (output power $50 \mathrm{~mW}$ ). To achieve optimal exposure for image capturing as well as to avoid heating effects of illuminated plate the optical attenuator was used enabling 3-5 $\mathrm{mW} / \mathrm{cm}^{2}$ power density of the scattered laser light on the whole agar plate surface. Main components of the system are presented in Fig. 1. The speckle images with 10 MPix resolution were captured by a CMOS camera with different frame rates, for different experiments: 1 frame per 2, 5, 6, 10, 20, or $30 \mathrm{sec}$, and different durations of experiments about 10-25 h. The white light LED illumination was used for acquisition of RGB images of growing bacteria colonies. Further RGB images was used for referential visual determination of bacteria colonies.

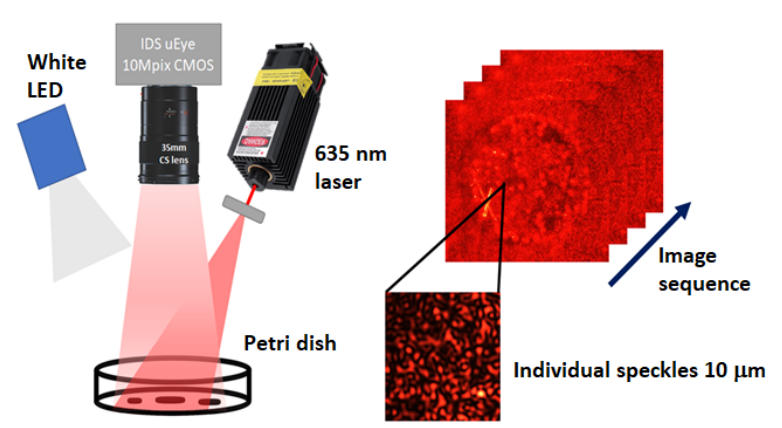

Fig. 1 Setup scheme for burst image capturing of bacteria growing process under $635 \mathrm{~nm}$ laser and white LED illumination.

\subsubsection{Evaluation of colony growth under white light illumination}

Microbial growth on the agar surface and formation of colony is a self-limiting process which starts with the maximum rate, which is gradually inhibited by exhaustion of necessary nutrients, accumulation of end products of microbial metabolism. The most common mathematical model describing microbial colony growth is the Gompertz model, which mathematical expression is presented in Eq. (1).

$$
\mu(t)=\mu \cdot e^{-\mu_{i}\left(t-t_{0}\right)}
$$

where $\mu$ is specific growth rate $\left(\mathrm{h}^{-1}\right), \mu_{0}$ - specific growth rate at beginning of colony formation $\left(\mathrm{h}^{-1}\right), \mu_{i}$ - specific growth rate at infliction (or specific inhibitory rate), $t_{0}$ represents time at beginning of the colony growth and $t$ is the actual time $(\mathrm{h})$.

$$
r(t)=r_{\text {max }} \cdot e^{-\left(\ln \left(\frac{r_{\max }}{r_{0}}\right) \cdot e^{-\left(\mu_{i} \cdot\left(t-t_{0}\right)\right)}\right)} .
$$

Eq. (2) shows the growth of the colony radius, where $r_{\text {max }}$ is the maximal radius that the colony can reach in the given environmental conditions, $r_{0}$ is the initial radius at $t_{0}, \mu_{i}$ is the specific growth rate at the inflexion point of the area curve. Over time gradual inhibition of colony growth occurs, which can be described by $\mu_{i}$. To use these equations to simulate growth of real CFU, $\mu_{0}$ and $\mu_{i}$ should be found. A specific set of parameters should be found to describe microbial colony growth using the Gompertz model. It was done experimentally for each microbial species and cultivation conditions (temperature and media). Fast growing sea bacteria Vibrio natriegens were chosen for the experiments. Bacteria were grown on salt rich, agar plates in room temperature (see the materials and methods section). First, the series of micro images were used to find the growth rate at the beginning of CFU formation (when colony growth rate is practically equal to $\left.\mu_{0}\right)$. Thus, $\mu_{0}$ for $V$. natriegens in the room temperature was determined approximately $3.875 \pm 0.25 \mathrm{~h}^{-1}$. Also, a series of CFU images under white light were taken to experimentally find the specific $V$. natriegens $\mathrm{CFU}$ inhibitory rate $\left(\mu_{i}\right)$. For this purpose, 
the growth of the $V$. natriegens $\mathrm{CFU}$ was recorded (one image in $15 \mathrm{~min}$, from 0 to $20 \mathrm{~h}$ or more) to estimate the growth of the colony radius under white light illumination. The diameter of each CFU was determined from images using the ImageJ program. The CFU was identified from images by visual inspection (without photo enhancing). Due to the low cell concentrations, the contrast of RGB images under white light illumination was low and CFU diameters were determined not earlier than after 8-12 h. Colony diameters were determined from the image series over time. Using Eq. (2) the $\mu_{i}$ for $V$. natriegens were calculated at given temperature and media conditions. Example of obtained results under white light illumination presented in Fig. 4 (white light experiments curves 1-4).

$$
R M S E=\sqrt{\frac{1}{n} \sum_{i=1}^{n}\left(R_{\text {Gompertz }}[i]-R_{\text {measured }}[i]\right)^{2}} .
$$

According to the experimentally obtained colony results, we set maximum colony radius to be 170 pix and initial colony radius $\left(r_{0}\right) 0.1$ pix ( 1 pix equals $5 \mu \mathrm{m}$ ). When simulating $\mu_{i}$ for $V$. natriegens in the room temperature for the given colony growth, the $\mu_{i}$ was $0.11 \mathrm{~h}^{-1}$. However, $\mu_{i}$ is not a fixed number. This coefficient characterizes decreasing of the colony growth rate over the time. The colony growth rate is dependent on many factors such as: temperature, number of surrounding colonies, and local concentration gradient of nutrients. Usually, the $\mu_{i}$ can vary by $20-50 \%$ among the same organism. To extract parameters from the given experimental data set, parameter scan was used. As a criterion we used Root Mean Square Error (RMSE) to minimum Eq. (3) to find $\mu_{i}$ and $t_{0}$. Where $R_{\text {Gompertz }}$ is the radius estimated from the Gompertz model, $R_{\text {measured }}$ is the radius determined experimentally, $n$ is the number of measurement points. RMSE values can vary in each experiment as they represent a match between theoretical and experimental curves.

\subsubsection{Evaluation of colony growth by the processing of laser speckle time series images}

For the analysis of speckle images, the growth of the $V$. natriegens colony under laser light illumination was recorded. The time interval between subsequent frames was $30 \mathrm{sec}$, which corresponds to a sampling frequency of $33.3 \mathrm{mHz}$. The frequency domain of the observed signal is up to $16.7 \mathrm{mHz}$. According to our previous research described in paper $[12,13]$ "signal" (or time signal) represents the result of the correlation and interpolation algorithm between each pair of consecutive speckle image frames. Further, the scatter of the specific growth rate $\mu_{i}$, with the average experimental value of $0.17 \mathrm{~h}^{-1}$ (Fig. 3) was calculated. To obtain a curve representing growth of colony radius the following steps on processing of speckle images were performed: 1) the location of the colonies was manually marked and automatically divided into $20 \times 20$ pix sections; 2) converting each $20 \times 20$ pix section into a time series; 3 ) determination of signal threshold and rising time at low frequencies according to Eq. (4):

$$
\operatorname{spectrogram}[k, f]=|\operatorname{STFT}[k, f]|^{2},
$$

where $\operatorname{STFT}[k, f]$ is the Fourier transform of $\operatorname{sig}[n] \cdot w[n-k]$, a complex function representing the phase and magnitude of the signal over time and frequency; 4) marking and determination of the times and spatial locations of detected signals; 5) marking the growth start times of each $20 \times 20$ pix square, knowing the location of each square in space and its distance from the colony center (marked manually in step 1), finally the colony radius was calculated as a function of time (Fig. 3, red line). In general, the above-mentioned processing approach determines time events that characterize dynamic changes in speckle signals for all spatial sections $(20 \times 20$ pix each) throughout the whole experimental data set.

\subsection{Detection and processing of NIR autofluorescence under $785 \mathrm{~nm}$ laser excitation}

The spectral measurements were conducted by an experimental spectroscopic system for analysis of AF emission under $785 \mathrm{~nm}$ laser excitation. The experimental setup comprises a diode laser (785 nm, $100 \mathrm{~mW}, \mathrm{CW})$, a USB 4000 portable spectrometer $(3.5 \mathrm{~nm}$ resolution, Ocean Optics, Inc., Dunedin, USA), $400 \mu \mathrm{m}$ fiber-optic probe, and a computer (Fig. 2). To block the reflected laser light in-line filter holder with $810 \mathrm{~nm}$ long pass filter was used. The diameter of laser spot on the measurement surface (skin, cuvette, samples) was approximately $4 \mathrm{~mm}^{2}$. Experimental setup was used for detection of AF emission from cells cultures, ex vivo formalized skin lesion samples and in vivo skin.

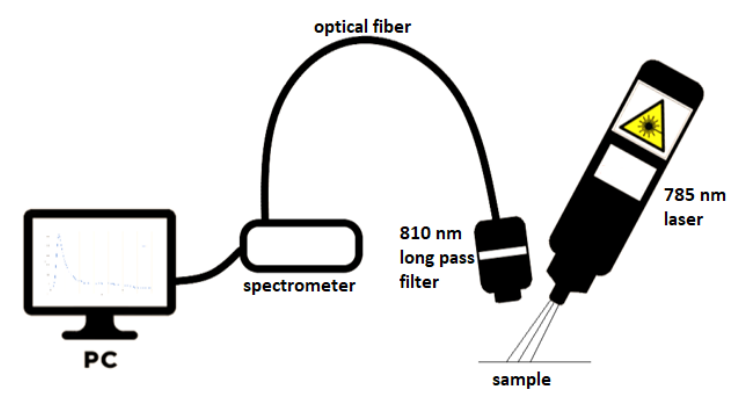

Fig. 2 Experimental setup for detection and analysis of NIR AF under $785 \mathrm{~nm}$ continuous laser excitation. Setup comprises $\mathrm{CW}$ laser with output power $100 \mathrm{~mW}$, optical probe with $810 \mathrm{~nm}$ long pass filter (OD $>4)$, optical fiber, spectrometer, and computer.

For cells experiments each cell culture (b16f10 and DC-3F) line was cultivated in growth DMEM (SigmaAldrich, St. Louis, USA) medium containing 10\% fetal bovine serum (Sigma-Aldrich, St. Louis, MO, USA), 1\% L-glutamine solution (Invitrogen Inc., Carlsbad, CA, 
USA). The cells were grown in $96 \mathrm{~mm}$ culture dish (TPP, Trasadingen, Switzerland) at $37{ }^{\circ} \mathrm{C}$ in humidified $5 \%$ $\mathrm{CO}_{2}$ atmosphere in the incubator. After trypsinization, cells were suspended in medium $(0.25 \mathrm{M}$ glucose, $1 \mathrm{mM}$ $\mathrm{MgCl}_{2}, 10 \mathrm{mM} \mathrm{Na}_{2} \mathrm{HPO}_{4}(\mathrm{pH} 7.4)$ ) at a concentration of $\sim 3 \mathrm{Mio}$ cells $/ \mathrm{ml}$. After the preparation, the cells suspension was filled to the quartz cuvette (filling volume $3.5 \mathrm{ml}$, pathlength $1 \mathrm{~cm}$ ) for further detection of NIR AF emission. The detection of NIR AF emission was performed separately for each cell culture suspension. Spectrometer integration time for collection of AF and dark signal was set to $10 \mathrm{sec}$. Dark signal was acquired from the media $(0.25 \mathrm{M}$ glucose, $1 \mathrm{mM} \mathrm{MgCl} 2,10 \mathrm{mM}$ $\mathrm{Na}_{2} \mathrm{HPO}_{4}$ (pH 7.4)) containing no cells. The distance between fiber probe and measurement cuvette was set to $4 \mathrm{~mm}$, the diameter of the laser spot on cuvette surface was $4 \mathrm{~mm}^{2}$.

For the detection of AF spectra from formalinized ex vivo skin lesion samples the $5 \mathrm{sec}$ integration time was used. For each ex vivo sample, three spectra from the lesion area and three spectra from the surrounding healthy skin were collected. Further, all collected spectra were averaged and divided to the two groups: pigmented lesions and healthy skin. As result, the averaged spectra of pigmented lesions and healthy skin were depicted in the graph. In total, 10 ex vivo samples were included in the study: 3 melanomas, 3 dysplastic nevi and 4 dermal nevi.

For the in vivo experiments the same experimental setup was used. The spectra were collected from the outside part of fingertips where melanin concentration is higher. In total, 3 healthy 30-40 years old persons with different skin phototypes were participated in the study. To minimize intensity fluctuations three AF spectra were collected on the outside of fingertips and further averaged. Averaged spectra were normalized to the AF maximum value at $825 \mathrm{~nm}$. According to the geometry of experimental setup the laser spot diameter on the skin surface was approximately $4 \mathrm{~mm}^{2}$, providing optical density $\sim 250 \mathrm{~mW} / \mathrm{cm}^{2}$. To avoid painful effects caused by heating the laser exposition time was minimized to $1 \mathrm{sec}$.

\section{Results and discussion}

\subsection{Microbial growth analysis}

Fig. 3 shows the colony radius growth as a function of time for speckle image obtained according to the Gompertz model (Eg. 2). Using the scanning method for several parameters (growth rate, growth start time, maximum radius of colony) it is possible to get the most optimal suitable Gompertz curve in which the Root Mean Square Error value will be minimal. The numerous experiments were performed using speckle imaging technique and image analysis under white light illumination (see results in Fig. 4) to understand the typical signal dynamics of $V$. natriegens colony growth.

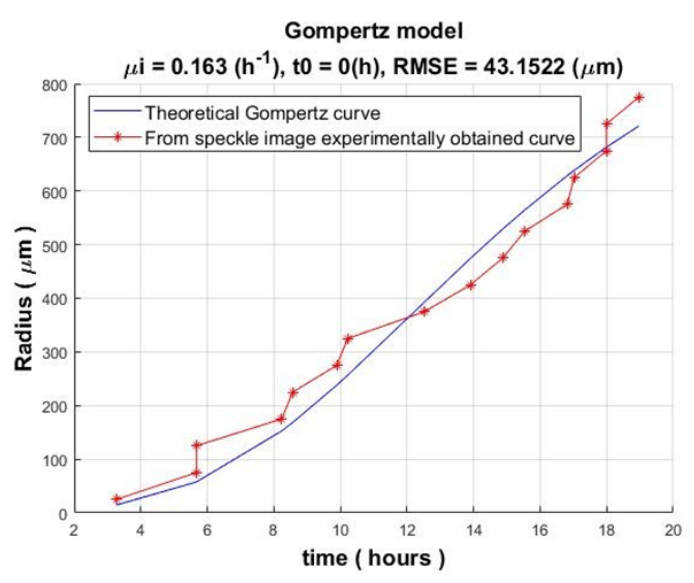

Fig. $3 V$. natriegens colony radius obtained by speckle image experiment (red) and theoretical Gompertz curve (blue).

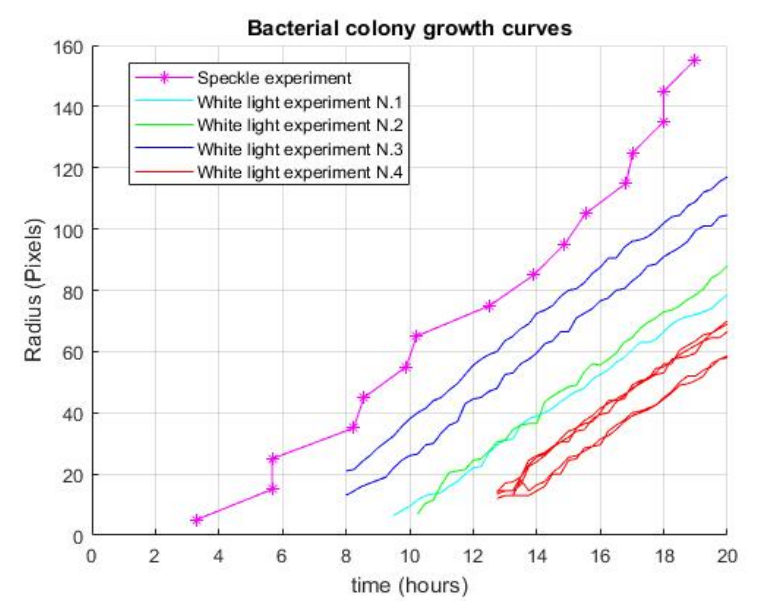

Fig. 4 Comparison of growth curves of a colony of bacteria $V$. natriegens measured by analysis of laser speckle images and visual examination under white light illumination.

Comparing these two (laser speckle and white light imaging) analyses techniques, it was obtained that with the speckle imaging technique it is possible to detect microbial activity earlier than using white light imaging. The colony formation on the agar media was observed $3 \mathrm{~h}$ after bacterial inoculation, while the CFU growth of the colony in white light could be detected after 8-13 h. All signals depicting CFU growth (obtained from speckle and white light images) demonstrate similar dynamics (the graphs of radius increase over time are parallel to each other). However, the growth signal graphs are scattered across $O X$-axes, which can be attributed to fluctuated room temperature across the experiments thus affecting growth speed and colony detection. To conclude, the proposed method based on analysis of laser speckle time series images provides earlier responses of growing bacteria in comparison with classical CFU growth detection under white light illumination. The results (growing curves) demonstrate wide distribution which can be attributed to unstable ambient temperature, which fluctuated across the experiments and therefore altered growth speed. Undoubtedly, further additional 
studies performed in a controlled microbiological environment are necessary.

\subsection{Analysis of NIR autofluorescence under $785 \mathrm{~nm}$ laser excitation}

The practical use of NIR AF for tissue characterization and diagnostics has a great scientific interest. Moreover, the exact determination of endogenous fluorophores responsible for NIR AF emission is still under discussion. In this paper, in collaboration with Ekaterina Borisova, we have conducted series of experiments in different conditions to find spectral features suitable for skin diagnostics. NIR AF experiments were performed at invitro cells cultures, at in vivo skin and formalinized skin lesion ex vivo samples.

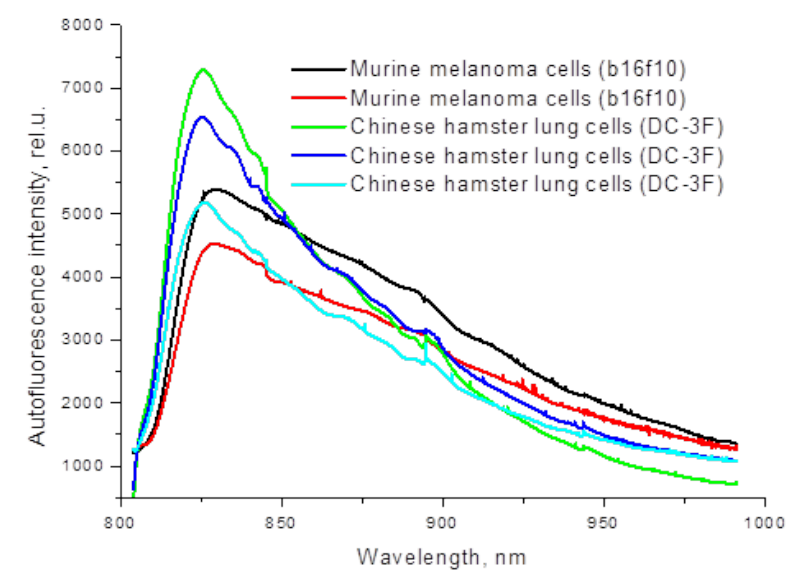

Fig. 5 NIR AF spectra obtained from melanocytic (murine melanoma cells) and melanocytic (Chinese hamster lung cells) cells under $785 \mathrm{~nm}$ laser excitation.

NIR AF spectra obtained from two different types of cells is presented in Fig. 5. As shown, all obtained AF spectra has a wide intensity distribution, and can be characterized by main maxima at $\sim 825 \mathrm{~nm}$.

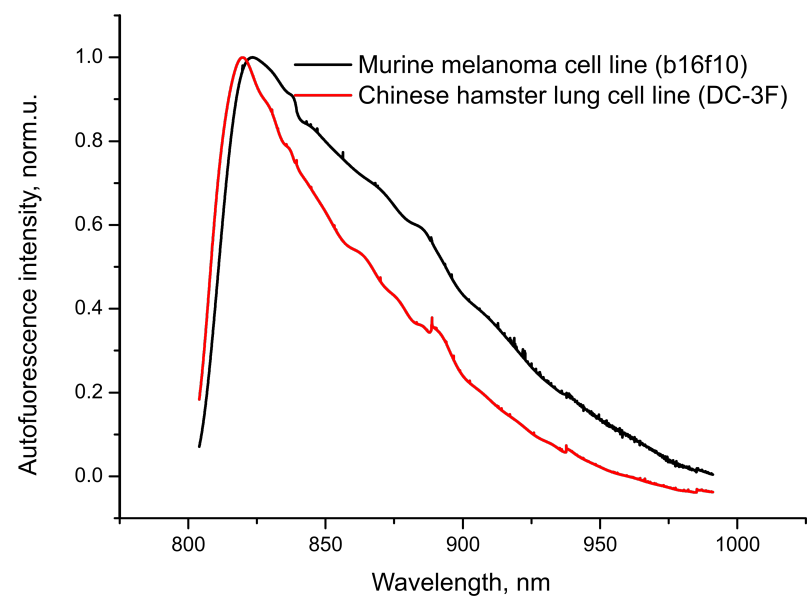

Fig. 6 Normalized NIR AF spectra of murine melanoma and Chinese hamster lung cells under $785 \mathrm{~nm}$ laser excitation.
In general, the AF spectra of highly pigmented melanoma cells can be described by the lower AF relative intensity in comparison with lung cells. After the spectra normalization (Fig. 6) the significant differences in shape of the spectra become more visible. In particular, the normalized averaged AF spectrum of melanoma cells demonstrates higher integral square in comparison with lung cells spectrum. Further we have examined AF in vivo spectra acquired from healthy volunteers with different skin photo-types. The Fig. 7 represents NIR AF spectra collected on the top of the finger where the melanin concentration is higher. The obtained spectra demonstrate higher AF intensity in volunteers with phototype III in comparison with skin phototype II. Remarkably, that spectra acquired from cell cultures (Fig. 5) demonstrates an opposite trend melanocytic cells had a lower AF intensity in comparison with melanocytic cells.

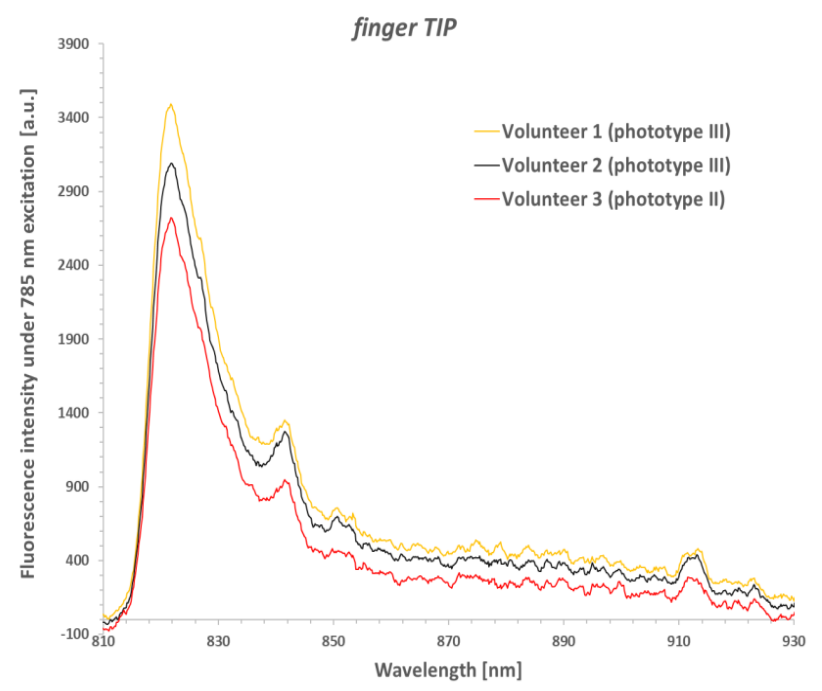

Fig. 7 Averaged autofluorescence spectra for 3 different volunteer's fingertips in vivo.

Moreover, all collected in vivo NIR AF (Fig. 7) spectra can be characterized by three emission maxima: main peak at $\sim 825 \mathrm{~nm}$, and two smaller peaks located at $\sim 840 \mathrm{~nm}$ and $\sim 850 \mathrm{~nm}$. The local peak at $\sim 910 \mathrm{~nm}$ most probably is attributed to the CCD etaloning effects and could be neglected for the analysis. Obtained AF spectral features (peaks at $840 \mathrm{~nm}$ and $850 \mathrm{~nm}$ ) are in a good agreement with available literature data, fully described by I. Bratchenko et al. [7] Normalization (Fig. 8) of AF spectra acquired from healthy in vivo skin demonstrates that all processed spectra are nearly equal. No spectral shift of emission maxima appeared. However, the intensity variations of AF peaks at $840 \mathrm{~nm}$ and $850 \mathrm{~nm}$ can be observed. In particular, the intensity of skin phototype II has a lowest AF intensity at $840 \mathrm{~nm}$. Undoubtedly, the number of collected signals is statistically very low, as well as relative concentration of melanin content in skin phototypes III and II does not provide strong scientific evidence to draw any conclusions. However, the obtained data is in quantitative agreement with earlier published papers available in literature [7-11]. 


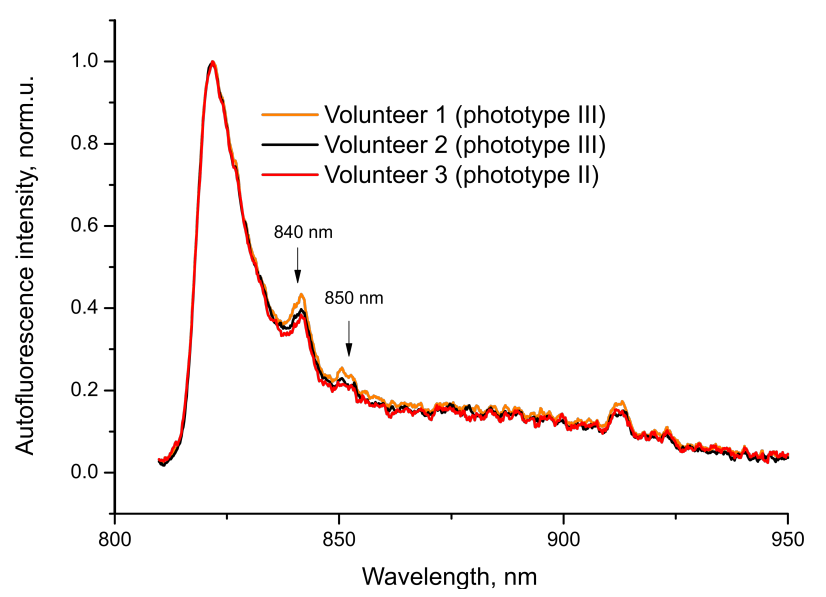

Fig. 8 Normalized AF spectra for 3 different volunteer's fingertips in vivo.

Further in collaboration with Ekaterina Borisova at the Institute of Electronics, Bulgarian Academy of Sciences we have collected NIR AF signals from formalinized ex vivo skin lesion samples (Fig. 6). The obtained spectra were divided into two groups: 1) NIR AF spectra from melanocytic lesions and 2) spectra obtained from surrounding healthy skin.

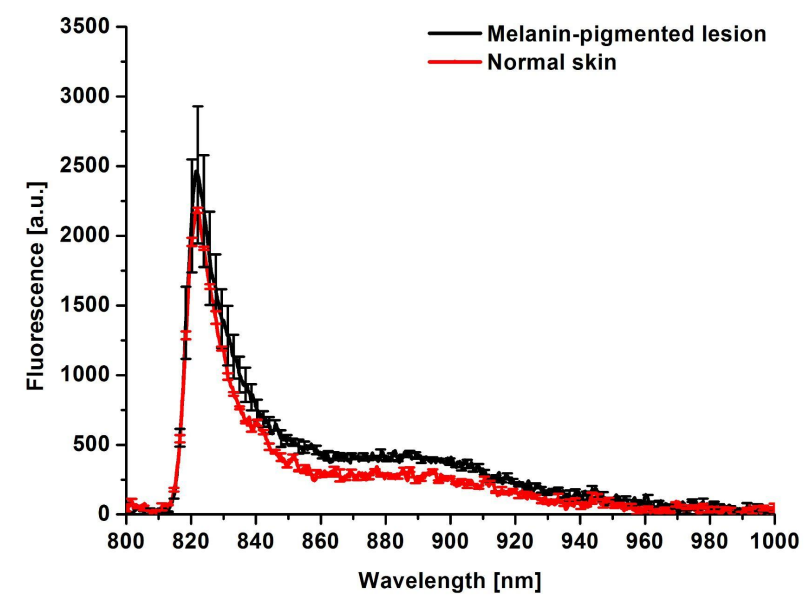

Fig. 9 NIR AF spectra collected from ex vivo skin lesion samples under $785 \mathrm{~nm}$ laser excitation.

Obtained averaged spectra also demonstrates (Fig. 9) the same trend acquired in in vivo skin the highly pigmented melanocytic lesions have a higher AF intensity in comparison with healthy skin. The shape of the formalinized spectra differs in comparison with spectra collected in vivo. In particular, the emission peaks of $\mathrm{AF}$ at $\sim 840 \mathrm{~nm}$ and $\sim 850 \mathrm{~nm}$ were not observed in ex vivo samples. Probably, it can be attributed to the dehydration of the skin samples due to its formalization. Also, the destruction of the responsible fluorophores under influence of formalin is not excluded. Notably, the integral spectral square of the melanocytic lesions has is higher in comparison with healthy skin. The same tendency has been acquired in cell cultures $\mathrm{AF}$ spectra (Fig. 6).
To summarize, in presented paper we have analyzed NIR AF spectra obtained at different conditions. The acquired spectral shapes of melanocytic cells and ex vivo lesions have a similar spectral feature. In particular, the integral square of the melanoma cells and melanocytic lesions is higher in comparison with healthy skin and amelanocytic lung cells. The relative intensities at AF emission maxima $(820 \mathrm{~nm})$ does not provide strong correlation with tissue and cells experiments, and most probably could not be used for diagnostics purpose. However, the analysis of AF spectra at the spectral regions between $840-900 \mathrm{~nm}$ seems to be potential for assessment of melanocytic lesions and melanoma diagnostics. Moreover, the development of imaging techniques intended for visualization of suspicious areas of the skin should be implemented at spectral bands including AF maxima $(825 \mathrm{~nm})$ and regions between $840 \mathrm{~nm}$ and $900 \mathrm{~nm}$. Undoubtedly, the future studies complemented with hypotheses and theoretical models are necessary to understand the exact phenomenon of the NIR AF.

\section{Conclusions}

To conclude the presented paper demonstrates a potential of laser applications for evaluation of microbial activity and skin lesion diagnostics. Analysis of laser speckle time series images provides an earlier response of growing bacteria in comparison with classical CFU growth detection under white light illumination.

Obtained AF spectra under $785 \mathrm{~nm}$ laser excitation deliver a quantitative correlation between in vitro melanoma cells and ex vivo melanocytic lesions. The analysis of AF intensity at spectral maxima does not provide strong correlation with tissue and cells melanin content. Further studies should be focused on investigation of in vivo tissue and native histology samples in order to identify key morphological features responsible for NIR AF spectral variations.

\section{Disclosures}

All authors declare that there is no conflict of interests in this paper.

\section{Funding}

This research is funded by the European Regional Development Fund project "Fast and cost effective machine learning based system for microbial growth analysis" (agreement No: 1.1.1.1/19/A/147).

\section{Acknowledgments}

This article is dedicated to Ekaterina Borisova, whose contribution in science we greatly appreciate and respect. In the short time we spent doing experiments together, we found that Ekaterina was also a passionate lover of Latvian ballet. Unfortunately, we did not manage to organize a joint ballet visit to Latvia, which we had planned. May she rest in peace. 


\section{References}

1. J. Yoon, K. Lee, and Y. Park, "A simple and rapid method for detecting living microorganisms in food using laser speckle decorrelation," arXiv:1603.07343 (2016).

2. H. Loutfi, F. Pellen, B. Le Jeune, R. Lteif, M. Kallassy, G. Le Brun, and M. Abboud, "Real-time monitoring of bacterial growth kinetics in suspensions using laser speckle imaging," Scientific Reports 10(1), 408 (2020).

3. H. Kim, A. K. Singh, A. K. Bhunia, and E. Bae, "Laser-induced speckle scatter patterns in Bacillus colonies," Frontiers in Microbiology 5, 537 (2014).

4. H. C. Grassi, L. C. García, M. L. Lobo-Sulbarán, A. Velásquez, F. A. Andrades-Grassi, H. Cabrera, J. E. AndradesGrassi, and E. D. J. Andrades, "Quantitative laser biospeckle method for the evaluation of the activity of trypanosoma cruzi using VDRL plates and digital analysis," PLoS Neglected Tropical Diseases 10(12), e0005169 (2016).

5. H. C. Grassi, A. Velásquez, O. M. Belandria, M. L. Lobo-Sulbarán, J. E. Andrades-Grassi, H. Cabrera, and E. D. Andrades, "Biospeckle laser digital image processing for quantitative and statistical evaluation of the activity of Ciprofloxacin on Escherichia coli K-12,” Laser Physics 29(7), 075603 (2019).

6. K. Zhou, C. Zhou, A. Sapre, J. H. Pavlock, A. Weaver, R. Muralidharan, J. Noble, J. Kovac, Z. Liu, and A. Ebrahimi, "Dynamic laser speckle imaging meets machine learning to enable rapid antibacterial susceptibility testing (DyRAST)," ACS Sensors 5(10), 3140-3149 (2020).

7. I. A. Bratchenko, Y. A. Khristoforova, L. A. Bratchenko, A. A. Moryatov, S. V. Kozlov, E. G. Borisova, and V. P. Zakharov, "Optical Biopsy of Amelanotic Melanoma with Raman and Autofluorescence Spectra Stimulated by 785 nm Laser Excitation,” Journal of Biomedical Photonics \& Engineering 7(2), 020308 (2021).

8. Z. Huang, H. Lui, D. I. McLean, M. Korbelik, and H. Zeng, "Raman spectroscopy in combination with background near-infrared autofluorescence enhances the in vivo assessment of malignant tissues," Photochemistry and Photobiology 81(5), 1219-1226 (2005).

9. E. G. Borisova, I. A. Bratchenko, Y. A. Khristoforova, L. A. Bratchenko, T. I. Genova, A. I. Gisbrecht, A. A. Moryatov, S. V. Kozlov, P. P. Troyanova, and V. P. Zakharov, "Near-infrared autofluorescence spectroscopy of pigmented benign and malignant skin lesions," Optical Engineering 59(6), 061616 (2020).

10. S. Wang, J. Zhao, H. Lui, Q. He, and H. Zeng, "In vivo near-infrared autofluorescence imaging of pigmented skin lesions: methods, technical improvements and preliminary clinical results," Skin Research and Technology 19(1), 20-26 (2013).

11. M. Saif, W. J. Kwanten, J. A. Carr, I. X. Chen, J. M. Posada, A. Srivastava, J. Zhang, Y. Zheng, M. Pinter, S. Chatterjee, S. Softic, C. R. Kahn, K. van Leyen, O. T. Bruns, R. K. Jain, and M. G. Bawendi, "Non-invasive monitoring of chronic liver disease via near-infrared and shortwave-infrared imaging of endogenous lipofuscin," Nature Biomedical Engineering 4(8), 801-813 (2020).

12. I. Balmages, J. Liepins, S. Zolins, D. Bliznuks, I. Lihacova, and A. Lihachev, "Laser speckle imaging for early detection of microbial colony forming units," Biomedical Optics Express 12(3), 1609-1620 (2021).

13. I. Balmages, D. Bliznuks, J. Liepins, S. Zolins, and A. Lihachev, "Laser speckle time-series correlation analysis for bacteria activity detection," Proceedings of SPIE 11359, 113591D (2020). 